\title{
Erratum
}

\section{Decay of Solutions of the Wave Equation in the Kerr Geometry}

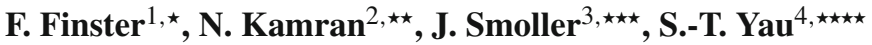 \\ 1 NWF I - Mathematik, Universität Regensburg, 93040 Regensburg, Germany. \\ E-mail: Felix.Finster@mathematik.uni-regensburg.de \\ 2 Department of Math. and Statistics, McGill University, Montréal, Québec, Canada H3A 2K6. \\ E-mail: nkamran@math.McGill.CA \\ 3 Mathematics Department, The University of Michigan, Ann Arbor, MI 48109, USA. \\ E-mail: smoller@umich.edu \\ 4 Mathematics Department, Harvard University, Cambridge, MA 02138, USA. \\ E-mail: yau@math.harvard.edu
}

Received: 4 April 2006 / Accepted: 30 November 2007

Published online: 18 March 2008 - (C) Springer-Verlag 2008

Commun. Math. Phys. 264, 465-503 (2006)

As was recently pointed out to us by Thierry Daudé, there is an error on the last page of our paper [2]. Namely, the inequality (8.3) cannot be applied to the function $\Psi(t)$ because it does not satisfy the correct boundary conditions. This invalidates the last two inequalities of the paper, and thus the proof of decay is incomplete. We here fill the gap using a different method. At the same time, we will clarify in which sense the sum over the angular momentum modes converges in [1, Theorem 1.1] and [2, Theorem 7.1], an issue which in these papers was not treated in sufficient detail. The arguments in these papers certainly yield weak convergence in $L_{\text {loc }}^{2}$; here we will prove strong convergence.

Our method here is to split the wave function into the high and low energy components. For the high energy component, we show that the $L^{2}$-norm of the wave function can be bounded by the energy integral, even though the energy density need not be everywhere positive (Sect. 1). For the low energy component we refine our ODE techniques (Sect. 2). Combining these arguments with a Sobolev estimate and the Riemann-Lebesgue lemma completes the proof (Sect. 3).

We begin by considering the integral representation of [2, Theorem 7.1], for fixed $k$ and a finite number $n_{0}$ of angular momentum modes,

$$
\Phi^{n_{0}}(t, r, \vartheta):=\int_{-\infty}^{\infty} d \omega e^{-i \omega t} \hat{\Phi}^{n_{0}}(\omega, r, \vartheta)
$$

The online version of the original article can be found under doi:10.1007/s00220-006-1525-8.

\footnotetext{
^ Research supported in part by the Deutsche Forschungsgemeinschaft.

$\star \star$ Research supported by NSERC grant \# RGPIN 105490-2004.

$\star \star \star$ Research supported in part by the NSF, Grant No. DMS-010-3998.

$\star \star \star \star$ Research supported in part by the NSF, Grant No. 33-585-7510-2-30.
} 
where for notational convenience we have omitted the $\varphi$-dependence (i.e. the factor $\left.e^{-i k \varphi}\right)$, and $\hat{\Phi}^{n_{0}}(\omega)$ is defined by

$$
\hat{\Phi}^{n_{0}}(\omega, r, \vartheta)=\frac{1}{2 \pi} \frac{1}{\omega \Omega} \sum_{n=1}^{n_{0}} \sum_{a, b=1}^{2} t_{a b}^{\omega n} \Phi_{\omega n}^{a}(r, \vartheta)<\Psi_{\omega n}^{b}, \Psi_{0}>
$$

(as in [1], we always denote the scalar wave function by $\Phi$, whereas $\Psi=\left(\Phi, \partial_{t} \Phi\right)$ is a two-component vector). We recall that for large $\omega$, the WKB-estimates of [1, Sect. 6] ensure that the fundamental solutions $\Phi_{k \omega n}^{b}$ go over to plane waves, and thus, since the initial data $\Psi_{0}$ is smooth and compactly supported, the function $\hat{\Phi}^{n_{0}}(\omega, r, \vartheta)$ decays rapidly in $\omega$ (for details on this method see [3, proof of Theorem 6.5]). As a consequence, $\Phi^{n_{0}}$ and its derivatives are, for $r$ and $\vartheta$ in any compact set, uniformly bounded in time. Our goal is to obtain estimates uniform in $n_{0}$, justifying that, as $n_{0} \rightarrow \infty, \Phi^{n_{0}}$ converges in $L_{\text {loc }}^{2}$ to the solution of the wave equation.

To arrange the energy splitting we choose for a given parameter $J>0$, a positive smooth function $\chi_{\mathrm{H}+}$ supported on $(J, \infty)$ with $\left.\chi_{\mathrm{H}+}\right|_{[2 J, \infty)} \equiv 1$. We define $\chi_{\mathrm{H}-}$ by $\chi_{\mathrm{H}-}(\omega)=\chi_{\mathrm{H}+}(-\omega)$ and set $\chi_{\mathrm{L}}=1-\chi_{\mathrm{H}+}-\chi_{\mathrm{H}-\text {. }}$. We introduce the high-energy contributions $\hat{\Phi}_{\mathrm{H} \pm}^{n_{0}}$ and the low-energy contribution $\hat{\Phi}_{\mathrm{L}}^{n_{0}}$ by

$$
\hat{\Phi}_{\mathrm{H} \pm}^{n_{0}}(\omega, r, \vartheta)=\chi_{\mathrm{H} \pm}(\omega) \hat{\Phi}^{n_{0}}(\omega, r, \vartheta), \quad \hat{\Phi}_{\mathrm{L}}^{n_{0}}(\omega, r, \vartheta)=\chi_{\mathrm{L}}(\omega) \hat{\Phi}^{n_{0}}(\omega, r, \vartheta) .
$$

\section{1. $L^{2}$-Estimates of the High-Energy Contribution}

We recall from $[1,(2.5)]$ that the energy density of a wave function $\Phi$ in the Kerr geometry is given by

$$
\begin{aligned}
\mathcal{E}(\Phi)= & \left(\frac{\left(r^{2}+a^{2}\right)^{2}}{\Delta}-a^{2} \sin ^{2} \vartheta\right)\left|\partial_{t} \Phi\right|^{2}+\Delta\left|\partial_{r} \Phi\right|^{2} \\
& +\sin ^{2} \vartheta\left|\partial_{\cos \vartheta} \Phi\right|^{2}+\left(\frac{1}{\sin ^{2} \vartheta}-\frac{a^{2}}{\Delta}\right) k^{2}|\Phi|^{2} .
\end{aligned}
$$

Note that the energy density need not be positive due to the last term. However, the next theorem shows that the energy integral

$$
E(\Phi):=\int_{r_{1}}^{\infty} d r \int_{-1}^{1} d \cos \vartheta \mathcal{E}(\Phi(t))
$$

(which is independent of time due to energy conservation), in the high-energy region is both positive and can be bounded from below by the $L^{2}$-norm. In what follows, we only consider $\Phi_{\mathrm{H}+}^{n_{0}}$ because $\Phi_{\mathrm{H}-}^{n_{0}}$ can be treated similarly.

Theorem 1.1. There exists a positive constant $J_{0}$ (depending only on $k$, but independent of $n_{0}$ and $\Psi_{0}$ ), such that for all $J \geq J_{0}$ the following inequality holds for every $t$ :

$$
E\left(\Phi_{H+}^{n_{0}}\right) \geq \frac{J^{2}}{2} \int_{r_{1}}^{\infty} \frac{\left(r^{2}+a^{2}\right)^{2}}{\Delta} d r \int_{-1}^{1} d \cos \vartheta\left|\Phi_{H+}^{n_{0}}(t)\right|^{2} .
$$

The remainder of this section is devoted to the proof of Theorem 1.1. We begin with the following lemma. 
Lemma 1.2. Let $g, \Phi$ be measurable functions, $g$ real and $\Phi$ complex, such that $\Phi$ and $g \Phi$ are in $L^{1}(\mathbb{R})$. Then

$$
\int_{\mathbb{R}} d \omega \int_{\mathbb{R}} d \omega^{\prime} \min \left(g(\omega), g\left(\omega^{\prime}\right)\right) \Phi(\omega) \overline{\Phi\left(\omega^{\prime}\right)} \geq \inf g\left|\int_{\mathbb{R}} \Phi\right|^{2} .
$$

Proof. Using a standard approximation argument, it suffices to consider the case that $g$ and $\Phi$ are simple functions of the form

$$
g(\omega)=\sum_{a=1}^{A} g_{a} \chi\left(K_{a}\right), \quad \Phi(\omega)=\sum_{a=1}^{A} \Phi_{a} \chi\left(K_{a}\right)
$$

where $\chi\left(K_{a}\right)$ is the characteristic function of the set $K_{a}$, and $\left(K_{a}\right)_{a=1, \ldots, A}$ forms a partition of $\mathbb{R}$. Then the above inequality reduces to

$$
\sum_{a, b=1}^{A} \min \left(g_{a}, g_{b}\right) \Phi_{a}\left|K_{a}\right| \overline{\Phi_{b}}\left|K_{b}\right| \geq \min g \sum_{a, b=1}^{A} \Phi_{a}\left|K_{a}\right| \overline{\Phi_{b}}\left|K_{b}\right| \text {. }
$$

In the case $A=2$ and $g_{1} \leq g_{2}$, this inequality follows immediately from the calculation

$$
\begin{aligned}
g_{1}\left|c_{1}\right|^{2}+g_{1}\left(c_{1} \overline{c_{2}}+\overline{c_{1}} c_{2}\right)+g_{2}\left|c_{2}\right|^{2} \geq & g_{1}\left|c_{1}\right|^{2}+g_{1}\left(c_{1} \overline{c_{2}}+\overline{c_{1}} c_{2}\right) \\
& +g_{1}\left|c_{2}\right|^{2}=g_{1}\left|c_{1}+c_{2}\right|^{2},
\end{aligned}
$$

where $c_{a}:=\Phi_{a}\left|K_{a}\right|$. In the case $A=3$ and $g_{1} \leq g_{2} \leq g_{3}$, we get

$$
\begin{aligned}
& g_{1}\left(\left|c_{1}\right|^{2}+2 \operatorname{Re}\left(c_{1} \overline{c_{2}}+c_{1} \overline{c_{3}}\right)\right)+g_{2}\left(\left|c_{2}\right|^{2}+2 \operatorname{Re}\left(c_{2} \overline{c_{3}}\right)\right)+g_{3}\left|c_{3}\right|^{2} \\
& \geq g_{1}\left(\left|c_{1}\right|^{2}+2 \operatorname{Re}\left(c_{1} \overline{c_{2}}+c_{1} \overline{c_{3}}\right)\right)+g_{2}\left|c_{2}+c_{3}\right|^{2} \geq g_{1}\left|c_{1}+c_{2}+c_{3}\right|^{2} .
\end{aligned}
$$

The general case is similar.

The next lemma bounds the $L^{2}$-norm of $\Phi_{\mathrm{H}+}^{n_{0}}$ and its partial derivatives by a constant depending on $n_{0}$ and $\Psi_{0}$.

Lemma 1.3. There is a constant $C=C\left(n_{0}, \Psi_{0}\right)$ such that for every $t$,

$$
\int_{r_{1}}^{\infty} \frac{\left(r^{2}+a^{2}\right)^{2}}{\Delta} d r \int_{-1}^{1} d \cos \vartheta\left(\left|\Phi_{H+}^{n_{0}}(t)\right|^{2}+\left|\partial_{r} \Phi_{H+}^{n_{0}}(t)\right|^{2}+\sum_{k=1}^{3}\left|\partial_{t}^{k} \Phi_{H+}^{n_{0}}(t)\right|^{2}\right) \leq C .
$$

Proof. It suffices to consider one angular momentum mode. For notational simplicity we omit the angular dependence. Since $\Phi_{\mathrm{H}+}^{n_{0}}$ and its derivatives are locally pointwise bounded uniformly in time, it follows that their $L^{2}$-norms on any compact set are bounded in time.

Near the event horizon, we work with the fundamental solution $\phi$ in the ReggeWheeler variable $u$ (see $[1,(2.18,5.2)]$ and $[2$, Sect. 3]). Then for any sufficiently small $u_{0}$, the integral of $|\Phi|^{2}$ over the region $u<u_{0}$ can be written as

$$
\int_{-\infty}^{u_{0}} d u|\phi|^{2}
$$


where it is now convenient to write our integral representation (1) in the form

$$
\phi(t, u)=\int_{-\infty}^{\infty} d \omega\left(h_{+}(\omega) \dot{\phi}_{\omega}(u)+h_{-}(\omega) \overline{\dot{\phi}_{\omega}(u)}\right) e^{-i \omega t} .
$$

Here the functions $h_{ \pm}$have rapid decay and, as they are supported away from the set $\left\{0, \omega_{0}\right\}$, they are also smooth (see [2, Sect. 3.1]). Using the Jost representation $[2,(3.7,3.10)]$, the function $\phi(t, u)$ can be decomposed as

$$
\phi(t, u)=\phi_{+}(t, u)+\phi_{-}(t, u)+\rho(t, u),
$$

where

$$
\begin{aligned}
& \phi_{ \pm}(t, u):=\int_{-\infty}^{\infty} d \omega h_{ \pm}(\omega) e^{ \pm i\left(\omega-\omega_{0}\right) u-i \omega t}, \\
& |\rho(t, u)| \leq C e^{\gamma u} \text { for all } t, \text { where } C, \gamma>0 .
\end{aligned}
$$

Note that the smoothness of $h_{ \pm}$implies that $\phi_{ \pm}$decay rapidly in $u$. From the exponential decay of the factor $e^{\gamma u}$ it is obvious that the $L^{2}$-norm of $\rho$ is bounded uniformly in $t$. The $L^{2}$-norms of $\phi_{ \pm}$can be estimated as

$$
\int_{-\infty}^{u_{0}}\left|\phi_{ \pm}(t, u)\right|^{2} d u \leq \int_{-\infty}^{\infty}\left|\phi_{ \pm}(t, u)\right|^{2} d u=\int_{-\infty}^{\infty}\left|\phi_{ \pm}\left(0, u^{\prime}\right)\right|^{2} d u^{\prime}=: c
$$

where $u^{\prime}=u \mp t$.

Near infinity, we work similarly with the fundamental solutions $\grave{\phi}[2,(3.2)]$. Again using the Jost representation [2, (3.15) and Lemma 3.3], we get terms depending only on $t \pm u$ as well as error terms which decay like $1 / u$ and are thus in $L^{2}$.

The time derivatives can be treated in the same way, since a time derivative merely gives a factor of $\omega$ which can be absorbed into $h_{ \pm}$. For the spatial derivatives we use similarly the estimates for the first derivatives of the Jost functions.

Our next step is to decompose the energy integral into a convenient form. To do this, we introduce a positive mollifier $\alpha \in C_{0}^{\infty}([-1,1])$ with the properties $\alpha(-\omega)=\alpha(\omega)$ and $\int \alpha(\omega) d \omega=1$. We define the function $\Gamma\left(\omega-\omega^{\prime}\right)$ by mollifying the Heaviside function $\Theta$,

$$
\Gamma\left(\omega-\omega^{\prime}\right)=(\Theta * \alpha)\left(\omega-\omega^{\prime}\right) .
$$

We now substitute the Fourier representation of $\Phi_{\mathrm{H}+}^{n_{0}}$ into the formula for the energy density (2). For simplicity we omit the indices $n_{0}$ and $H+$ in what follows. Omitting the first positive summand in (2), we get the inequality

$$
\begin{aligned}
& \mathcal{E}(\Phi)(t, r, \vartheta) \geq \int_{-\infty}^{\infty} d \omega \int_{-\infty}^{\infty} d \omega^{\prime} e^{-i\left(\omega-\omega^{\prime}\right) t} \\
& \times\left\{\left(\frac{1}{\sin ^{2} \vartheta}-\frac{a^{2}}{\Delta}\right) k^{2} \hat{\Phi}(\omega) \overline{\hat{\Phi}\left(\omega^{\prime}\right)}\right. \\
& \quad+\Gamma\left(\omega-\omega^{\prime}\right)\left(\Delta \partial_{r} \hat{\Phi}(\omega) \overline{\partial_{r} \hat{\Phi}\left(\omega^{\prime}\right)}+\sin ^{2} \vartheta \partial_{\cos \vartheta} \hat{\Phi}(\omega) \overline{\partial_{\cos \vartheta} \hat{\Phi}\left(\omega^{\prime}\right)}\right) \\
& \left.\quad+(1-\Gamma)\left(\omega-\omega^{\prime}\right)\left(\Delta \partial_{r} \hat{\Phi}(\omega) \overline{\partial_{r} \hat{\Phi}\left(\omega^{\prime}\right)}+\sin ^{2} \vartheta \partial_{\cos \vartheta} \hat{\Phi}(\omega) \overline{\partial_{\cos \vartheta} \hat{\Phi}\left(\omega^{\prime}\right)}\right)\right\}
\end{aligned}
$$


We multiply by a positive test function $\eta(u) \in C_{0}^{\infty}(\mathbb{R})$ and integrate over $r$ and $\cos \vartheta$. Integrating by parts in (5) and (6) to the right and left, respectively, we can use the wave equation

$$
\begin{aligned}
& {\left[-\frac{\partial}{\partial r} \Delta \frac{\partial}{\partial r}-\frac{1}{\Delta}\left(\left(r^{2}+a^{2}\right) \omega+a k\right)^{2}\right.} \\
& \left.-\frac{\partial}{\partial \cos \vartheta} \sin ^{2} \vartheta \frac{\partial}{\partial \cos \vartheta}+\frac{1}{\sin ^{2} \vartheta}\left(a \omega \sin ^{2} \vartheta+k\right)^{2}\right] \hat{\Phi}(\omega)=0
\end{aligned}
$$

to obtain

$$
\begin{gathered}
\int_{r_{1}}^{\infty} d r \int_{-1}^{1} d \cos \vartheta \eta(u)((5)+(6))=\int_{r_{1}}^{\infty} d r \int_{-1}^{1} d \cos \vartheta \int_{-\infty}^{\infty} d \omega \int_{-\infty}^{\infty} d \omega^{\prime} e^{-i\left(\omega-\omega^{\prime}\right) t} \\
\times\left\{-\eta^{\prime}(u)\left(r^{2}+a^{2}\right)\left(\Gamma \hat{\Phi}(\omega) \overline{\partial_{r} \hat{\Phi}\left(\omega^{\prime}\right)}+(1-\Gamma) \partial_{r} \hat{\Phi}(\omega) \overline{\left.\hat{\Phi}\left(\omega^{\prime}\right)\right)}\right.\right. \\
\left.+\eta(u)\left(\Gamma g\left(\omega^{\prime}\right)+(1-\Gamma) g(\omega)\right) \hat{\Phi}(\omega) \overline{\hat{\Phi}\left(\omega^{\prime}\right)}\right\}
\end{gathered}
$$

where

$$
g(\omega, r, \vartheta)=\frac{1}{\Delta}\left(\left(r^{2}+a^{2}\right) \omega+a k\right)^{2}-\frac{1}{\sin ^{2} \vartheta}\left(a \omega \sin ^{2} \vartheta+k\right)^{2},
$$

and we used that

$$
\frac{d}{d r} \eta(u)=\eta^{\prime}(u) \frac{r^{2}+a^{2}}{\Delta} .
$$

We interchange the orders of integration of the spatial and frequency integrals and let $\eta$ tend to the constant function one. In the term corresponding to (4), Lemma 1.3 allows us to pass to the limit. In $(7,8)$ the situation is a bit more involved due to the factors of $\Gamma$. However, since multiplication by $\Gamma\left(\omega-\omega^{\prime}\right)$ corresponds to convolution with its Fourier transform $\check{\Gamma}(t)$, we can again apply Lemma 1.3 and pass to the limit using Lebesgue's dominated convergence theorem. To make this method more precise, let us show in detail that the expression

$$
\int_{r_{1}}^{\infty} d r \int_{-1}^{1} d \cos \vartheta \int_{-\infty}^{\infty} d \omega \int_{-\infty}^{\infty} d \omega^{\prime} e^{-i\left(\omega-\omega^{\prime}\right) t}(1-\eta(u)) \Gamma\left(\omega-\omega^{\prime}\right) g\left(\omega^{\prime}\right) \hat{\Phi}(\omega) \overline{\hat{\phi}\left(\omega^{\prime}\right)}
$$

tends to zero as $\eta$ converges to the constant function one. Rewriting the factor $\Gamma$ with a time convolution, we obtain the expression

$$
\int_{-\infty}^{\infty} d \tau \check{\Gamma}(\tau) F(\tau)
$$

where $F$ and $\check{\Gamma}$ are defined by

$$
\begin{aligned}
& F(\tau)=\int_{r_{1}}^{\infty} d r \int_{-1}^{1} d \cos \vartheta(1-\eta(u)) \Phi(t-\tau, r, \vartheta)(\check{g} * \bar{\Phi})(t-\tau, r, \vartheta), \\
& \check{\Gamma}(\tau)=\frac{1}{2 \pi} \int_{-\infty}^{\infty} \Gamma(b) e^{-i b \tau} d b .
\end{aligned}
$$


Writing $g$ as a polynomial in $\omega$,

$$
g(\omega)=g_{0}+g_{1} \omega+g_{2} \omega^{2}
$$

where

$$
g_{0}=\frac{a^{2} k^{2}}{\Delta}-\frac{k^{2}}{\sin ^{2} \vartheta}, \quad g_{1}=2 a k\left(\frac{r^{2}+a^{2}}{\Delta}-1\right), \quad g_{2}=\frac{\left(r^{2}+a^{2}\right)^{2}}{\Delta}-a^{2} \sin ^{2} \vartheta,
$$

the function $\check{g} * \bar{\Phi}$ can be expressed explicitly in terms of $\bar{\Phi}$ and its time derivatives of order at most two. In order to compute $\check{\Gamma}$, we first note that the Fourier transform of the Heaviside function $\Theta$ is

$$
\check{\Theta}(\tau)=\frac{1}{2 \pi}\left(-i \frac{\mathrm{PP}}{\tau}+\pi \delta(\tau)\right),
$$

where "PP" denotes the principal part. Using (3) together with the fact that convolution in momentum space corresponds to multiplication in position space, we find that

$$
\check{\Gamma}(\tau)=\left(-i \frac{\mathrm{PP}}{\tau}+\pi \delta(\tau)\right) \check{\alpha}(\tau),
$$

where $\check{\alpha}$ is a Schwartz function with $\check{\alpha}(0)=(2 \pi)^{-1}$. According to Lemma 1.3 , the function $F$ is uniformly bounded,

$$
|F(\tau)| \leq \sup |\eta| \text { for all } \tau \in \mathbb{R}
$$

Using the rapid decay of $\check{\Gamma}$, we can for any given $\varepsilon$ choose a parameter $L>0$ such that

$$
\int_{\mathbb{R} \backslash[-L, L]}|\check{\Gamma}(\tau)||F(\tau)| \leq \varepsilon \sup |\eta| .
$$

On the interval $[-L, L]$, on the other hand, the singularity of $\hat{\Gamma}$ at $\tau=0$ can be controlled by at most first derivatives of $F$, and thus for a suitable constant $C=C(L)$,

$$
\left|\int_{-L}^{L} \check{\Gamma}(\tau) F(\tau)\right| \leq 2 C \sup _{[-L, L]}\left(|F|+\left|F^{\prime}\right|\right) .
$$

Using the rapid decay of $\Phi$ and its time derivatives in $u$, locally uniformly in $\tau$, we can make $\sup _{[-L, L]}\left(|F|+\left|F^{\prime}\right|\right)$ as small as we like. This shows that (9) really tends to zero as $\eta$ goes to the constant function one.

We conclude that

$$
\begin{aligned}
E\left(\Phi_{\mathrm{H}+}^{n_{0}}\right) \geq & \int_{r_{1}}^{\infty} d r \int_{-1}^{1} d \cos \vartheta \int_{-\infty}^{\infty} d \omega \int_{-\infty}^{\infty} d \omega^{\prime} e^{-i\left(\omega-\omega^{\prime}\right) t} \\
\times & \left\{\left(\min \left(g(\omega), g\left(\omega^{\prime}\right)\right)+\frac{k^{2}}{\sin ^{2} \vartheta}-\frac{a^{2} k^{2}}{\Delta}\right) \hat{\Phi}(\omega) \overline{\hat{\Phi}\left(\omega^{\prime}\right)}\right. \\
& \left.+\left(\Gamma g\left(\omega^{\prime}\right)+(1-\Gamma) g(\omega)-\min \left(g(\omega), g\left(\omega^{\prime}\right)\right)\right) \hat{\Phi}(\omega) \overline{\hat{\Phi}\left(\omega^{\prime}\right)}\right\} .
\end{aligned}
$$


We apply Lemma 1.2 to obtain

$$
(14) \geq \int_{r_{1}}^{\infty} d r \int_{-1}^{1} d \cos \vartheta\left(\inf _{\omega \geq J} g(\omega)+\frac{k^{2}}{\sin ^{2} \vartheta}-\frac{a^{2} k^{2}}{\Delta}\right)|\Phi(t, r, \vartheta)|^{2} .
$$

Using the explicit form of $g$, (10), we find that for sufficiently large $J$,

$$
(14) \geq \frac{J^{2}}{2} \int_{r_{1}}^{\infty} \frac{\left(r^{2}+a^{2}\right)^{2}}{\Delta} d r \int_{-1}^{1} d \cos \vartheta|\Phi(t, r, \vartheta)|^{2} .
$$

It remains to control the term (15). We write the $\omega, \omega^{\prime}$-integral of (15) in the form

$$
B:=\int_{-\infty}^{\infty} d \omega \int_{-\infty}^{\infty} d \omega^{\prime} e^{-i\left(\omega-\omega^{\prime}\right) t} h\left(\omega, \omega^{\prime}\right) \hat{\Phi}(\omega) \overline{\Phi\left(\omega^{\prime}\right)},
$$

where

$$
h\left(\omega, \omega^{\prime}\right)=\Gamma g\left(\omega^{\prime}\right)+(1-\Gamma) g(\omega)-\min \left(g(\omega), g\left(\omega^{\prime}\right)\right) .
$$

Introducing the variables $a=\frac{1}{2}\left(\omega+\omega^{\prime}\right)$ and $b=\frac{1}{2}\left(\omega-\omega^{\prime}\right)$, and using that $g(\omega)$ is a polynomial in $\omega$, a short calculation yields

$$
h(a+b, a-b)=\left(g_{1}+2 g_{2} a\right) S(2 b) \quad \text { where } \quad S(b):=b(\Theta(b)-\Gamma(b)) .
$$

Using $(12,13)$ together with the fact that the factor $b$ corresponds to a derivative in position space, we obtain

$$
\check{S}(\tau)=\frac{1}{2 \pi} \frac{d}{d \tau}\left(\frac{1-2 \pi \check{\alpha}(\tau)}{\tau}\right)=-\frac{d}{d \tau} \int_{0}^{1} \alpha^{\prime}(s \tau) d s .
$$

This is a smooth function which decays quadratically at infinity; in particular, it is integrable.

We thus obtain for the Fourier transform of $h$ the explicit formula

$$
\begin{aligned}
\check{h}\left(\tau, \tau^{\prime}\right) & =\frac{1}{(4 \pi)^{2}} \int_{-\infty}^{\infty} d \omega \int_{-\infty}^{\infty} d \omega^{\prime} h\left(\omega, \omega^{\prime}\right) e^{-i\left(\omega t-\omega^{\prime} t^{\prime}\right)} \\
& =\left(g_{1} \delta\left(\tau-\tau^{\prime}\right)+2 i g_{2} \delta^{\prime}\left(\tau-\tau^{\prime}\right)\right) \check{S}\left(\frac{\tau+\tau^{\prime}}{2}\right) .
\end{aligned}
$$

Using Plancherel for distributions, we obtain

$$
\begin{aligned}
B= & \int_{-\infty}^{\infty} d \tau \int_{-\infty}^{\infty} d \tau^{\prime} \check{h}\left(\tau, \tau^{\prime}\right) \Phi(t-\tau) \overline{\Phi\left(t-\tau^{\prime}\right)} \\
= & \int_{-\infty}^{\infty} d \tau \check{S}(\tau)\left[g_{1} \Phi(t-\tau) \overline{\Phi(t-\tau)}\right. \\
& \left.\quad+i g_{2}\left(\partial_{t} \Phi(t-\tau) \overline{\Phi(t+\tau)}-\Phi(t-\tau) \overline{\partial_{t} \Phi(t+\tau)}\right)\right] .
\end{aligned}
$$

Integrating over space, we can use the explicit formulas for $g_{1}$ and $g_{2}$ and apply Lemma 1.3 to obtain

$$
(15)=\int_{r_{1}}^{\infty} d r \int_{-1}^{1} d \cos \vartheta B \geq-C\left(n_{0}, \Psi_{0}\right) \int_{-\infty}^{\infty}|\check{S}(\tau)| d \tau .
$$


We now let $\alpha$ tend to the Dirac delta; then $\check{\alpha}$ tends to the constant function $(2 \pi)^{-1}$. As a consequence, the $L^{1}$-norm of $\breve{S}$ tends to zero, and thus (15) becomes positive in this limit. Hence the energy is bounded from below by (16). This concludes the proof of Theorem 1.1.

\section{Pointwise Estimates for the Low-Energy Contribution}

The low-energy contribution can be written as

$$
\Phi_{\mathrm{L}}^{n_{0}}(t, r, \vartheta)=\frac{1}{2 \pi} \sum_{n=1}^{n_{0}} \int_{-\infty}^{\infty} \frac{d \omega}{\omega \Omega} e^{-i \omega t} \chi_{\mathrm{L}}(\omega) \sum_{a, b=1}^{2} t_{a b}^{\omega n} \Phi_{\omega n}^{a}(r, \vartheta)<\Psi_{\omega n}^{b}, \Psi_{0}>
$$

We now derive pointwise estimates for the large angular momentum modes.

Theorem 2.1. For any $u_{0}<u_{1}$ there is a constant $C>0$ such that for all $\omega \in(-2 J, 2 J) \backslash\left\{\omega_{0}, 0\right\}$ and for all $u, u^{\prime} \in\left(u_{0}, u_{1}\right)$,

$$
\sum_{n=1}^{\infty}\left|\frac{1}{\Omega} \sum_{a, b=1}^{2} t_{a b} \phi^{a}(u) \phi^{b}\left(u^{\prime}\right)\right|<C .
$$

Proof. From [2, Sect. 5] the coefficients $t_{a b}$ have the explicit form

$$
T:=\left(t_{a b}\right)=\left(\begin{array}{cc}
1+\operatorname{Re} \frac{\alpha}{\beta} & -\operatorname{Im} \frac{\alpha}{\beta} \\
-\operatorname{Im} \frac{\alpha}{\beta} & 1-\operatorname{Re} \frac{\alpha}{\beta}
\end{array}\right),
$$

where the transmission coefficients $\alpha$ and $\beta$ are defined by

$$
\grave{\phi}=\alpha \dot{\phi}+\beta \bar{\phi}
$$

and $\phi_{1}=\operatorname{Re} \dot{\phi}, \phi_{2}=\operatorname{Im} \dot{\phi}$. The estimates of [2, Sect. 4.3] are obviously valid for $\omega$ in any bounded set; in particular for $\omega \in(-2 J, 2 J) \backslash\left\{\omega_{0}, 0\right\}$. We use these estimates in what follows, also using the same notation. We choose $n_{1}$ so large that $u_{+}<u_{0}$, and thus on the whole interval $\left(u_{0}, 2 u_{1}\right)$ the invariant disk estimates of Lemmas 4.2 and 4.8 in [2] hold.

Rewriting the expression $t_{a b} \phi^{a} \phi^{b}$ with the Green's function (see the proof of Lemma 5.1 in [2]), this expression is clearly invariant under the phase transformation $\dot{\phi} \rightarrow e^{i \vartheta} \hat{\phi}$. Thus we can arrange that $\dot{\phi}\left(2 u_{1}\right)$ is real. Then the transmission coefficients are computed at $u=2 u_{1}$ by

$$
\left(\begin{array}{c}
\grave{\phi} \\
\dot{\phi}^{\prime}
\end{array}\right)=\left(\begin{array}{cc}
\dot{\phi} & \bar{\phi} \\
\dot{\phi}^{\prime} & \overline{\bar{\phi}^{\prime}}
\end{array}\right)\left(\begin{array}{l}
\alpha \\
\beta
\end{array}\right)=\dot{\phi}\left(\begin{array}{ll}
1 & 1 \\
\dot{y} & \bar{y}
\end{array}\right)\left(\begin{array}{l}
\alpha \\
\beta
\end{array}\right) \text {. }
$$

We thus obtain

$$
\alpha=-\left.\frac{\grave{\phi}}{2 \dot{\phi} \operatorname{Im} y}(\bar{y}-\grave{y})\right|_{u=2 u_{1}} \quad \beta=\left.\frac{\grave{\phi}}{2 \dot{\phi} \operatorname{Im} y}(\dot{y}-\grave{y})\right|_{u=2 u_{1}} .
$$


Hence

$$
\left|1+\frac{\alpha}{\beta}\right|=2 \frac{\mid \operatorname{Im} y ́}{|\dot{y}-\grave{y}|} \leq \frac{4|\Omega|}{\rho^{2} \operatorname{Re}(\dot{y}-\grave{y})},
$$

where all functions are evaluated at $u=2 u_{1}$, and where we used the relation

$$
\dot{\rho}^{2}=\frac{|\Omega|}{\operatorname{Im} y}
$$

which is an immediate consequence of $[2,(4.9)]$ and $w(\dot{\phi}, \bar{\phi})=2 i \Omega$. From $[2$, Lemma 4.6 and (4.42)] we know that for $\lambda$ sufficiently large,

$$
\operatorname{Re}(\dot{y}-\grave{y}) \geq \frac{1}{C} \text {. }
$$

Using the above formulas for $t_{a b}$, we conclude that

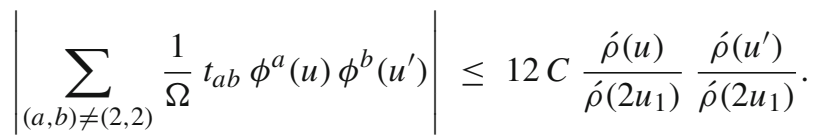

The argument after [2, (4.43)] shows that the two factors on the right decay like $\exp (-\sqrt{\lambda} / c)$.

It remains to consider the case $a=b=2$. Taking the imaginary part of the identity

$$
\dot{\phi}(u)=\dot{\phi}\left(2 u_{1}\right) \exp \left(\int_{2 u_{1}}^{u} \dot{y}\right)
$$

and using that $\dot{\phi}\left(2 u_{1}\right)$ is real, we find that

$$
\dot{\rho}(u)=\dot{\rho}\left(2 u_{1}\right) \exp \left(\int_{2 u_{1}}^{u} \operatorname{Re} \dot{y}\right)
$$

and thus

$$
\left|\phi_{2}(u)\right|=\dot{\rho}(u)\left|\sin \left(\int_{2 u_{1}}^{u} \operatorname{Im} \dot{y}\right)\right| \leq \rho(u) \int_{u}^{2 u_{1}} \operatorname{Im} \dot{y} .
$$

From $[2,(4.40)]$ we see that $\operatorname{Re} y$ is positive on $\left[u, 2 u_{1}\right]$. Using the relation $\rho^{\prime} / \rho=\operatorname{Re} y$, we conclude that $\rho$ is monotone increasing, and (18) yields that $\operatorname{Im} y ́$ is decreasing. Hence, again using (18),

$$
\left|\phi_{2}(u)\right| \leq \rho ́(u) \operatorname{Im} y ́(u)\left(2 u_{1}-u_{0}\right) \leq \sqrt{|\Omega| \operatorname{Im} y ́\left(u_{0}\right)}\left(2 u_{1}-u_{0}\right) .
$$

Using the above estimates for $\alpha / \beta$, we conclude that

$$
\left|\frac{1}{\Omega} t_{22} \phi^{2}(u) \phi^{2}\left(u^{\prime}\right)\right| \leq 3 \operatorname{Im} y\left(u_{0}\right)\left(2 u_{1}-u_{0}\right)^{2} .
$$

The invariant region estimate of Lemmas 4.2 and 4.8 in [2] yield that

$$
\operatorname{Im} y\left(u_{0}\right) \leq c|\Omega| \exp \left(-\frac{7}{8} \int_{u_{+}}^{u_{0}} \sqrt{V}\right),
$$

and we conclude that (19) again decays like $\exp (-\sqrt{\lambda} / c)$.

Since the eigenvalues $\lambda_{n}$ scale quadratically in $n$ [2, (2.11)], the summands in (17) decay exponentially in $n$. 
This theorem gives us pointwise control of the low-energy contribution, uniformly in time and in $n_{0}$, locally uniformly in space. To see this, we estimate the integral representation (1) by

$$
\left|\Phi_{\mathrm{L}}^{n_{0}}(t, r, \vartheta)\right| \leq \frac{1}{2 \pi} \sum_{n=1}^{n_{0}} \int_{-\infty}^{\infty} d \omega\left|\chi_{L}(\omega)\right| \sum_{a, b=1}^{2}\left|\frac{1}{\omega \Omega} t_{a b}^{\omega n} \Phi_{\omega n}^{a}(r, \vartheta)<\Psi_{\omega n}^{b}, \Psi_{0}>\right|
$$

In order to control the factor $\omega^{-1}$, we write the energy scalar product on the right in the form $[1,(2.15)]$, which involves an overall factor $\omega$. Now we can apply Theorem 2.1.

\section{Decay in $L_{\mathrm{loc}}^{\infty}$}

In this section we complete the proof of Theorem 1.1 in [2]. Let $K \subset\left(r_{1}, \infty\right) \times S^{2}$ be a compact set. The $L^{2}$-norm of $\Phi^{n_{0}}$ can be estimated by

$$
\left\|\Phi^{n_{0}}(t)\right\|_{L^{2}(K)} \leq\left\|\Phi_{\mathrm{H}+}^{n_{0}}(t)\right\|_{L^{2}(K)}+\left\|\Phi_{\mathrm{H}-}^{n_{0}}(t)\right\|_{L^{2}(K)}+\left\|\Phi_{\mathrm{L}}^{n_{0}}(t)\right\|_{L^{2}(K)} .
$$

According to Theorems 1.1 and 2.1, these norms are bounded uniformly in $n_{0}$ and $t$. Furthermore, our estimates imply that the sequence $\Phi^{n_{0}}(t)$ forms a Cauchy sequence in $L^{2}(K)$. To see this, we note that for any $n_{1}, n_{2}$,

$$
\left\|\Phi^{n_{1}}-\Phi^{n_{2}}\right\|_{L^{2}(K)} \leq E\left(\Phi_{\mathrm{H}+}^{n_{1}}-\Phi_{\mathrm{H}+}^{n_{2}}\right)+E\left(\Phi_{\mathrm{H}-}^{n_{1}}-\Phi_{\mathrm{H}-}^{n_{2}}\right)+\left\|\Phi_{\mathrm{L}}^{n_{1}}-\Phi_{\mathrm{L}}^{n_{2}}\right\|_{L^{2}(K)},
$$

uniformly in $t$. The energy terms on the right are the sums of the energies of the individual angular momentum modes. All the summands are positive due to Theorem 1.1, and thus the energy terms become small as $n_{1}, n_{2} \rightarrow \infty$. The same is true for the last summand due to our ODE estimates of Theorem 2.1. We conclude that $\Phi^{n_{0}}(t)$ converges in $L_{\text {loc }}^{2}$ as $n_{0} \rightarrow \infty$, and the limit coincides with the weak limit, which in [1,2] is shown to be the solution $\Phi(t)$ of the Cauchy problem.

To prove decay, given any $\varepsilon>0$ we choose $n_{0}$ such that $\left\|\Phi(t)-\Phi^{n_{0}}(t)\right\|_{L^{2}(K)}<$ $\varepsilon$ for all $t$. Since $\hat{\Phi}^{n_{0}}$ is continuous in $\omega$ and has rapid decay, uniformly on $K$, the Riemann-Lebesgue lemma yields that $\Phi^{n_{0}}(t)$ decays in $L^{\infty}(K) \subset L^{2}(K)$. Since $\varepsilon$ is arbitrary, we conclude that $\Phi(t)$ decays in $L^{2}(K)$.

Applying the same argument to the initial data $H^{n} \Psi_{0}$, we conclude that the partial derivatives of $\Phi(t)$ also decay in $L^{2}(K)$. The Sobolev embedding $H^{2,2}(K) \hookrightarrow L^{\infty}(K)$ completes the proof.

We wish to take the opportunity to correct a few other typos in the article: On page 476, line 12, the text "Proof of Theorem 3.1" should be replaced by "Proof of Theorem 3.2." On page 477 , line 10 , the two factors $\omega^{\mu}$ should be replaced by $\omega^{\mu-\frac{1}{2}}$. On page 477 , line 26 , the two factors $\pi u$ should be replaced by $\pi u \omega$. On page 477 , last line, the two factors $\omega^{\mu}$ should be replaced by $\omega^{\mu \frac{1}{2}}$. On page 490, equation (4.37), the summand $-\frac{T_{0}}{2}$ should be replaced by $-\frac{T_{0}}{2}|\Omega|$. On page 499 , line 12 , the function $\eta$ should be replaced by $\eta_{L}$. 


\section{References}

1. Finster, F., Kamran, N., Smoller, J., Yau, S.-T.: An integral spectral representation of the propagator for the wave equation in the Kerr geometry. Commun. Math. Phys. 260, 257-298 (2005)

2. Finster, F., Kamran, N., Smoller, J., Yau, S.-T.: Decay of Solutions of the Wave Equation in the Kerr Geometry. Commun. Math. Phys. 264, 465-503 (2006)

3. Kronthaler, J.: The Cauchy problem for the wave equation in the Schwarzschild geometry. J. Math. Phys. 47, 042501 (2006)

Communicated by G.W. Gibbons 